\title{
Kinematic Modeling and Analysis of a Walking Machine (Robot) Leg Mechanism on a Rough Terrain
}

\author{
Tesfaye O. Terefe ${ }^{1}$, Hirpa G. Lemu ${ }^{2 *}$, Addisu K/Mariam ${ }^{3}$,Tadele B. Tuli ${ }^{4}$ \\ 1 Mechanical Engineering Department Mizan-Tepi University, Mizan-Tepi, Ethiopia \\ 2 Faculty of Science and Technology, University of Stavanger, N-4036 Stavanger, Norway \\ 3 School of Mechanical Engineering, Jimma University, Ethiopia \\ 4 Addis Ababa Science and Technology University, Ethiopia \\ * Corresponding author's e-mail: Hirpa.g.lemu@uis.no
}

\begin{abstract}
Many manmade machines and mechanisms, including robots, function based on the concept of nature-inspired design, so that they can perform their intended duties by mimicking the working mechanisms of animals and insects. Accordingly, walking machines (robots) use wheels and tracks to cross rough terrain efficiently and in a more stable way than conventional robots. Legged walking robots in particular remain in a discontinuous contact with the ground that provides them with the capability to select routes to avoid obstacles or holes. This article reports a study conducted on kinematic modelling and analysis of a walking machine (robot) leg mechanism that can operate on rough terrain. Its kinematic mechanisms were analyzed using the Denavit-Hartenberg (DH) convention approach. Symbolic computations are also implemented to parametrically optimize the motion parameters of the robot leg mechanism. The equation of motion was derived from the dynamic analysis using the Euler-Lagrange method which involves kinetic and potential energy expressions. In order to validate the performance of the robot leg mechanism and motion behaviors, the kinematic motion analysis was performed in SolidWorks and MATLAB. The leg mechanism used is effective for rough terrain areas because it is capable of walking on the terrain with different amplitudes in terms of surface roughness and aerodynamics.
\end{abstract}

Keywords: walking machine, walking robot, kinematic analysis, motion analysis, Denavit-Hartenberg, rough terrain

\section{INTRODUCTION}

Recently, the interest in using robots that can mimic the natural motions of animals and insects to develop the robots that can improve productivity, safety, flexibility, controllability and accuracy, has significantly increased and is becoming more popular. Legged robot mechanisms in particular are nature-inspired models of cockroaches and insects [1]. With the support of powerful computers since the 1980s [2], in particular, the legged mechanisms of walking machines are being progressively developed because of their suitability for the applications that cannot be accomplished with tracked and wheeled walking machines.
Recent developments have facilitated not only the design of the mechanisms, but also enabled effective control and automation of the complex motions of the mechanisms $[3,4]$. Today's micro legged robots in their construction use the individual motor at each joint for actuation.

The mechanisms were analyzed by Reuleaux $[5,6]$ primarily in machine elements, by studying their combinations and exposed those laws of operation from the early science of machine kinematics. In its work of "Theoretische Kinematik" of 1875, Reuleaux [5, 6] offered many insights of discovering general acceptance and his second book "Lehrbuch der Kinematic" merged and extended earlier ideas, and philosophies in the study 
of mechanisms. His comprehensive and orderly views marked a high point in the improvement of kinematics, which is mostly dedicated to the investigation of machine elements.

In the one hundred years that followed Reuleaux, the contributions of scientists such as Hartmann, et al. [7] developed the science of constructing mechanisms to satisfy specific motions, namely, kinematic synthesis. The techniques they used were based on mechanics and geometry. It was not until 1940 that Svaboda [8] developed the numerical methods to design a simple but versatile mechanism known as four-bar linkage to generate the desired function using sufficient precision for engineering resolutions. The input to the crank indicates the values of the parameter of a function, and that on the output crank indicated the result of the function. Naturally, this four-bar linkage can generate only a partial amount of tasks because of the nature of the linkage itself. In early 1950s, the publication by Hrones and Nelson [9] of an "Atlas" containing approximately 10,000 coupler curves offered a very practical approach for the design engineers. This led to the progressive development of the kinematics of mechanisms as a popular area of study in engineering.

This paper focuses on study of kinematic synthesis and analysis of the leg mechanism in a walking robot for a rough terrain. The leg mechanism is modeled kinematically using integration of linkages with an objective of reducing the number of motors ensuring a design that minimizes the machine cost. In addition, the parametrically derived dimensional synthesis was carried out in a vector form using forward kinematic and inverse kinematic analysis.

\section{KINEMATIC ANALYSIS OF THE LEG MECHANISM OF A WALKING MACHINE}

The kinematic analysis of a leg mechanism is investigated based on the mechanism geometry and the known characteristics or kinematic quantities such as position, angular velocity, angular acceleration, that have a great importance in the design and analysis. In turn, the position and velocity give an insight into the functional behavior of the leg mechanism; the acceleration is related to the stresses and deformations in the leg components. The linkages are assumed to be fully rigid bodies for the kinematic analysis of the leg mechanism.

\section{Definition of related concepts}

In the past centuries, mechanisms have been configured into machines. Parallel with the development of kinematics of mechanisms as engineering science in the past forty years, regular terminologies and explanations were required to support its study. The definition of the concept mechanism by Reuleaux [5, 6], as an arrangement of inflexible or rigid bodies designed and coupled so that they can move up on each other with definite relative motion, is seen as the foundation of the understanding of mechanisms.

In the study of kinematics analysis of the walking machine leg mechanism, distinguishing the definitions and the roles of some terminologies such as links, linkages, frame, joints, as well as high and lower pairs are important. These terminologies are briefly explained below.

Links are the individual parts of the leg mechanism which is considered as a rigid body and linked with supplementary links to transfer motion and forces. In principle, a true rigid body does not change its shape during motion due to the strains in members of walking machines. In reality, true rigid body does not exist, it is an idealization used in mechanisms that do not consider small deflections or are designed to minimally deform and are considered as a rigid body. In literature, real machine member links are considered as a perfect rigid bodies for modeling purposes $[9,10]$.

A linkage is part of a mechanism where rigid body parts are connected together to form a chain. In a four-bar mechanism, for instance, a combination of a number of pair elements is connected by rigid pieces or links, where a pin or pivoted joints allow relative motion between their parts. In kinematic chains, linkages represent an assemblage of rigid bodies connected by kinematic joints of lower pairs, though both mechanisms and machines can be taken as a link. The term linkage is, in general, limited to kinematic chains made of lower pairs $[8,11]$.

A frame is a part which serves as the frame of reference for the motion of all parts. It is a typical part that does not exhibit motions. It is stationary or a fixed link in a leg mechanism, and when there is no link, it is actually a fixed link which determines the relative motion of other links. It is the reference from which all motions of the leg mechanisms are accounted for [8.12].

Joints are movable connections used to allow relative motions between links of the leg 
mechanism of a walking machine. Each joint reduces the mobility of the system. The joint between a crank and connecting rod of the slidercrank mechanism, for instance, is called a revolute joint or pin joint. The revolute joint has one DOF in that if one element is fixed, the revolute joint allows only the rotation of the other in a plane [8].

Lower and Higher Pairs: Connection between rigid bodies can be categorized as lower and higher pairs of elements. The gear and pinion which is used in the leg mechanism to transfer motion have a lower pair and a theoretical surface contact with one another, while the two elements in a higher pair joint have theoretically a point or a line contact. Lower pairs include the revolute or pin connections [9].

Any mechanical system can be classified according to its number of independent parameters which are needed to uniquely define the position in its space at any instant of time; i.e. degrees of freedom (DOF). The number of joints in a robot roughly translated to the DOF. In the design process, three different possibilities were considered. However, the up-down and forward-back motion is approximately linear and provides a method to propel forward or backward while adjusting to uneven terrain. For the leg mechanism of the walking machine, the general Gruebler-Kutzbach criterion can be applied to find the number of the DOF. This criterion is given as:

$$
D O F=6(n-1)-2 p-h
$$

where: $n$ - number of linkages,

$p$ - number of lower pairs and

$h$ - number of higher pairs

It is desired for a walking machine to have the flexibility required for walking on rough terrain while still being able to achieve fast locomotion and requiring minimal actuation for walking on flat terrain. For a walking machine to be capable of walking on various terrains, each leg requires three DOF to carry out the back and forth motion, up and down motion, and turning motion. Since the turning motion can be separated from other two motions in a leg mechanism, a two DOF planar mechanism, which provides the back and forth and the up and down motions are of interest. If all three DOF need to be simultaneously actuated for a rough terrain walking, then the walking machines can be slow. Conversely, if a leg mechanism is designed that only one DOF is required to be actuated for normal walking, then the speed of the walking machine can be fast.

\section{Direct kinematic analysis of a leg mechanism}

In earlier studies of mechanisms, two basic types of methods for analysis of mechanisms have mostly been used, namely; graphical and analytical. These methods involve different techniques for the analysis of mechanisms and they are suitable for a particular category of mechanisms. The graphical method is the classical approach conveniently used for simple mechanisms and provides better visualization. Nowadays, due to the developed sophisticated computer programs, some engineers in the design of mechanisms desire to work with the analytical approach.

When mechanisms are becoming more complex and many generalized coordinates are necessary, other types of analysis techniques are preferred, such as the Denavit-Hartenberg (DH) approach $[6,13,14]$, as well as computational tools, by developing the analysis algorithms. In walking machine construction, links are assumed as a rigid body and connected together by joints. When a walking machine leg mechanism is placed in a three-dimensional space, it has three positional DOFs and three orientation DOFs, i.e. a total of six. Denavit and Hartenberg [6] suggested that it is imaginable to use four parameters to achieve kinematic analysis of robots in multi-degree of freedom for the first time, in which links are connected by rotary or prismatic joints. This DH mechanism (depicted in Fig. 1) is used to represent and model the leg mechanism and drive its equation of motion. This representation is now used as a standard approach for the kinematic analysis of the walking machine leg mechanisms. It simplifies the ways of modeling the leg mechanism arrangement, irrespective of its order and difficulty or complexity $[15,16]$.

The significance of kinematic study overviews the presence of a technique which permits numerous locations of end-effector to be defined in a reliable and definite means. The DH concept technique says that each joint of the robot is assigned to a coordinate frame. Under this assumption, it is possible to simplify complex kinematic structures. In order to model the walking machine leg mechanism with DH representation [13] first, assign a local reference frame for every single joint i.e. assign a $Z$-axis and an $x$-axis. In DH representation, the $y$-axis cannot be used.

While performing the analysis of a walking machine by calculating the position, velocity and acceleration of points on the different parts of the 


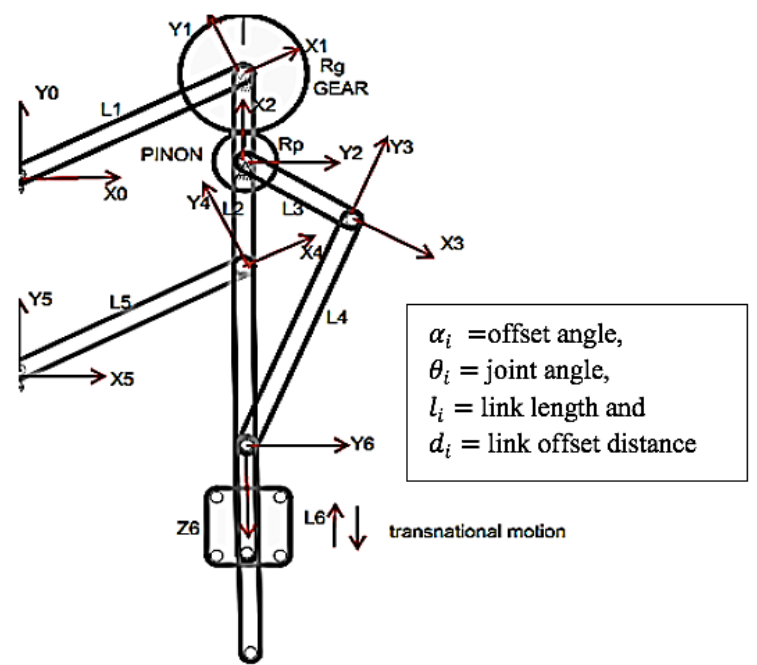

Fig. 1. DH convention of leg mechanism

leg mechanism and tracing the trajectory they follow are represented by homogeneous transformation matrices. Each homogenous transformation matrix is represented as a product of basic transformations, obtained for each link of the leg mechanism. For instance, in the DH representation in Fig. 1, the direct kinematics functions are constructed by decomposition of the individual transformations into a homogeneous transformation matrix.

Since the links lie on the same axis offset angle, the offset distance (excluding prismatic ones) between them is insignificant. The forward kinematic analysis is conducted to determine the position and orientation of the end points of the leg that touch the ground relative to the base frame of the walking robot. This is done in terms of the joint variables, which are the link extensions in the case of sliding or prismatic joint, and the angle between the links in the case of rotational or revolute joints.

Once the coordinate systems are rigidly fixed to each link of the leg mechanism and the link joint parameter is formed, coordinate transformation matrices are specified. The coordinate transformation matrices contain the information about the links and the displacements (both sliding and rotation) between the coordinate frames in the form of dual angles. Using the transformation matrix along the $\mathrm{z}$-axis, the overall transformation matrix is given by:

$$
[A]_{0}^{n}=[A]_{0}^{1}[A]_{1}^{2}[A]_{2}^{3}[A]_{3}^{4}[A]_{4}^{5}[A]_{5}^{n}
$$

where: $n$ is a number of joints.
Each of the homogeneous transformation matrices for all the joints, both the revolute joints (when $\mathrm{n}=1,2, \ldots 5$ ) and the prismatic joint (when $n=6$ ) are determined as follows.

When $\mathrm{n}=1$ : revolute joint - exhibits rotational motion and the homogeneous matrix $A_{0}^{1}$ is given as:

$$
A_{0}^{1}=\left[\begin{array}{cccc}
\cos \left(\theta_{1}\right) & -\sin \left(\theta_{1}\right) & 0 & a_{1} * \cos \left(\theta_{1}\right) \\
\sin \left(\theta_{1}\right) & \cos \left(\theta_{1}\right) & 0 & a_{1} * \sin \left(\theta_{1}\right) \\
0 & 0 & 1 & 0 \\
0 & 0 & 0 & 1
\end{array}\right]
$$

When $n=2$ revolute joint - exhibits rotational motion, and the homogenous matrix $A_{1}^{2}$ is given as:

$$
A_{1}^{2}=\left[\begin{array}{cccc}
\cos \left(\theta_{2}\right) & -\sin \left(\theta_{2}\right) & 0 & a_{2} * \cos \left(\theta_{2}\right) \\
\sin \left(\theta_{2}\right) & \cos \left(\theta_{2}\right) & 0 & a_{2} * \sin \left(\theta_{2}\right) \\
0 & 0 & 1 & 0 \\
0 & 0 & 0 & 1
\end{array}\right]
$$

In a similar fashion, the matrices for the rotational motion of the revolute joints when $n=3$, $\mathrm{n}=4$ and $\mathrm{n}=5$ are expressed as given in Eq. 5, 6 and 7 , respectively.

$$
\begin{aligned}
A_{2}^{3} & =\left[\begin{array}{cccc}
\cos \left(\theta_{3}\right) & -\sin \left(\theta_{3}\right) & 0 & a_{3} * \cos \left(\theta_{3}\right) \\
\sin \left(\theta_{3}\right) & \cos \left(\theta_{3}\right) & 0 & a_{3} * \sin \left(\theta_{3}\right) \\
0 & 0 & 1 & 0 \\
0 & 0 & 0 & 1
\end{array}\right] \\
A_{3}^{4} & =\left[\begin{array}{cccc}
\cos \left(\theta_{4}\right) & -\sin \left(\theta_{4}\right) & 0 & a_{4} * \cos \left(\theta_{4}\right) \\
\sin \left(\theta_{4}\right) & \cos \left(\theta_{4}\right) & 0 & a_{4} * \sin \left(\theta_{4}\right) \\
0 & 0 & 1 & 0 \\
0 & 0 & 0 & 1
\end{array}\right]
\end{aligned}
$$

$$
A_{4}^{5}=\left[\begin{array}{cccc}
\cos \left(\theta_{5}\right) & -\sin \left(\theta_{5}\right) & 0 & a_{5} * \cos \left(\theta_{5}\right) \\
\sin \left(\theta_{5}\right) & \cos \left(\theta_{5}\right) & 0 & a_{5} * \sin \left(\theta_{5}\right) \\
0 & 0 & 1 & 0 \\
0 & 0 & 0 & 1
\end{array}\right]
$$

When $n=6$, the mechanism has a prismatic joint that exhibits translational motion. Its homogenous matrix is then expressed as:

$$
A_{5}^{6}=\left[\begin{array}{cccc}
1 & 1 & 0 & 0 \\
0 & 1 & 0 & 0 \\
0 & 0 & 1 & d_{6} \\
0 & 0 & 0 & 1
\end{array}\right]
$$

As a result, the overall homogeneous transformation matrix defining the last link in touch 
with the ground with respect to the robot body is given by:

$$
[A]_{0}^{n}=\left[\begin{array}{cccc}
n_{x} & o_{x} & s_{x} & p_{x} \\
n_{y} & o_{y} & s_{y} & p_{y} \\
n_{z} & o_{z} & s_{z} & p_{z} \\
0 & 0 & 0 & 1
\end{array}\right]=\left[\begin{array}{cc}
A_{0}^{n} & P_{0}^{n} \\
0 & 1
\end{array}\right]
$$

where: $A_{0}^{n}=\left[\begin{array}{lll}n_{x} & o_{x} & s_{x} \\ n_{y} & o_{y} & s_{y} \\ n_{z} & o_{z} & s_{z}\end{array}\right]$ is the rotation matrix and

$$
P_{0}^{n}=\left[\begin{array}{l}
p_{x} \\
p_{y} \\
p_{z}
\end{array}\right] \text { is the position vector. }
$$

Both the position vector and the rotation matrix are functions of joint position $\theta$, and the leg tip position $\mathrm{p}_{\mathrm{x}}, \mathrm{p}_{\mathrm{y}}, \mathrm{p}_{\mathrm{z}}$ that can be directly obtained from the position vector $P_{0}^{n}$. The rotation matrix $A_{0}^{n}$ represents the orientation of the tip leg point, relative to the body of the robot.

The homogeneous transformation matrix for $\mathrm{n}$ number of joints can also be formulated as

$$
[A]_{0}^{n}=\left[\begin{array}{cccc}
n_{x} & o_{x} & s_{x} & p_{x} \\
n_{y} & o_{y} & s_{y} & p_{y} \\
n_{z} & o_{z} & s_{z} & p_{z} \\
0 & 0 & 0 & 1
\end{array}\right]
$$

\section{Inverse kinematic analysis of a leg mechanism}

In inverse kinematic modeling of mechanisms of the walking machine, the value of a joint position is determined in terms of the position and orientation of the tip leg point by employing inverse kinematics method. Thus, the homogeneous transformation matrix defining the walking leg with respect to the body of the walking machine is calculated as:

$$
\begin{gathered}
{[A]_{0}^{n}=\left[\begin{array}{cc}
A_{0}^{n} & P_{0}^{n} \\
0 & 1
\end{array}\right]=} \\
=\left[\begin{array}{llll}
a_{11} & a_{12} & a_{13} & a_{14} \\
a_{21} & a_{22} & a_{23} & a_{24} \\
a_{31} & a_{32} & a_{33} & a_{34} \\
a_{41} & a_{42} & a_{43} & a_{44}
\end{array}\right]
\end{gathered}
$$

Assuming $[A]_{0}^{n}=U_{0}$

$$
U_{0}=\left[\begin{array}{llll}
a_{11} & a_{12} & a_{13} & a_{14} \\
a_{21} & a_{22} & a_{23} & a_{24} \\
a_{31} & a_{32} & a_{33} & a_{34} \\
a_{41} & a_{42} & a_{43} & a_{44}
\end{array}\right]
$$

All elements of the matrix $U_{0}$ are known and given by a homogeneous transformation matrix. In fact, determining the joint position $\theta$ directly from the equation of the homogeneous transformation matrix is very difficult. Therefore, by successively pre-multiplying the two equations by the matrices $\left({ }^{j-1} A_{j}\right)^{-1}$, for $j=1, \ldots, n$, a new set of equations can be obtained.

$$
U_{0}=[A]_{0}^{n}=[A]_{0}^{1}[A]_{1}^{2}[A]_{2}^{3}[A]_{3}^{4}[A]_{4}^{5}[A]_{5}^{n}
$$

In a similar way, a new set of equations are given for $\mathrm{n}=6$ as:

$$
\begin{aligned}
& U_{1}=\left([A]_{0}^{1}\right)^{-1} U_{0}=[A]_{1}^{2}[A]_{2}^{3}[A]_{3}^{4}[A]_{4}^{5}[A]_{5}^{n} \\
& U_{2}=\left([A]_{1}^{2}\right)^{-1} U_{1}=[A]_{2}^{3}[A]_{3}^{4}[A]_{4}^{5}[A]_{5}^{n} \\
& U_{3}=\left([A]_{2}^{3}\right)^{-1} U_{2}=[A]_{2}^{3}[A]_{3}^{4}[A]_{4}^{5}[A]_{5}^{n} \\
& U_{4}=\left([A]_{3}^{4}\right)^{-1} U_{3}=[A]_{3}^{4}[A]_{4}^{5}[A]_{5}^{n} \\
& U_{5}=\left([A]_{4}^{5}\right)^{-1} U_{4}=[A]_{4}^{5}[A]_{5}^{n} \\
& U_{6}=\left([A]_{5}^{6}\right)^{-1} U_{5}=[A]_{5}^{n}
\end{aligned}
$$

where: $U_{j}=\left({ }^{j-1} \mathrm{~A}_{\mathrm{j}}\right)^{-1} \mathrm{U}_{\mathrm{j}-1}, \mathrm{j}=1, \ldots 6$, and these equations are named as forward equations.

By equating the position matrix from the homogeneous transformation matrix, the position of the tip of the leg touching the ground can be determined. This analytical approach considers the initial assumption that

$$
P_{0}^{n}=\left[\begin{array}{l}
P_{x}^{n} \\
P_{y}^{n} \\
P_{z}^{n}
\end{array}\right] \text { and }[R]_{0}^{n}=\left[\begin{array}{cc}
A_{0}^{n} & P_{0}^{n} \\
0 & 1
\end{array}\right]
$$

where: $R_{0}^{n}=\left[\begin{array}{lll}a_{11} & a_{12} & a_{13} \\ a_{21} & a_{22} & a_{23} \\ a_{31} & a_{32} & a_{33}\end{array}\right]$ and

$$
\begin{aligned}
& P_{0}^{n}=\left[\begin{array}{l}
P_{x}^{n} \\
P_{y}^{n} \\
P_{z}^{n}
\end{array}\right], \\
& \mathrm{R}_{0}^{\mathrm{n}}=\text { rotation matrix and } \\
& \mathrm{P}_{0}^{\mathrm{n}}=\text { position matrix } .
\end{aligned}
$$

\section{Instantaneous kinematic analysis of leg mechanism}

In order to derive the velocity relationships between the operational coordinates and the joint coordinates, the direct kinematic model that can express the linear velocities $\left(\mathrm{v}_{\mathrm{x}}, \mathrm{v}_{\mathrm{y}}, \mathrm{v}_{\mathrm{z}}\right)$ and 
angular velocities $\left(\mathrm{w}_{\mathrm{x}}, \mathrm{w}_{\mathrm{y}}, \mathrm{w}_{\mathrm{z}}\right)$ of the tool frame in terms of the Jacobian matrix and the derivatives of the joint variables, are obtained by differentiation with respect to time of the forward position kinematics equation.

$$
\dot{X}=\left[\begin{array}{c}
v_{x} \\
v_{y} \\
v_{z} \\
\omega_{x} \\
\omega_{y} \\
\omega_{z}
\end{array}\right]=\left[\begin{array}{ccc}
\frac{\partial f_{1}}{\partial \theta_{1}} & \cdots & \frac{\partial f_{1}}{\partial \theta_{n}} \\
\vdots & \ddots & \vdots \\
\frac{\partial f_{m}}{\partial \theta_{1}} & \cdots & \frac{\partial f_{m}}{\partial \theta_{n}}
\end{array}\right]\left(\begin{array}{c}
\dot{\theta}_{1} \\
\vdots \\
\vdots \\
\dot{\theta}_{n}
\end{array}\right)
$$

where: $n$ is the number of joints and this relation can simply be denoted as

$$
\dot{X}=J(\theta) \dot{\theta}
$$

where: $\dot{X}=\left[v_{x}, v_{y}, v_{z}, \omega_{x}, \omega_{y}, \omega_{z}\right]^{T} \quad$ denotes the vector of operational velocities, which is the velocity of the origin of the tool frame combined with its angular velocity with respect to the fixed Cartesian coordi-

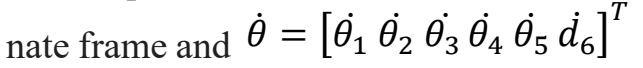
is the vector of angular velocities.

The $6 \times 6$ matrix $J(\theta)$ is the Jacobian matrix of the tool frame with respect to the base frame. The Jacobian matrix is composed of two parts: the upper half of the Jacobian $J_{v}(\theta)$ which is used to calculate the linear velocity $v$, and the lower half of the Jacobian $J_{\omega}(\theta)$, which calculates the angular velociy $\omega$. Thus, Eq. (15) can be rewritten as:

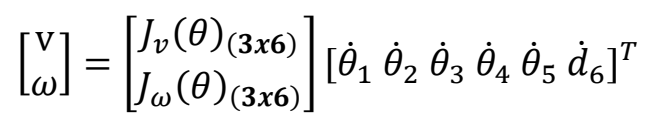

The elements of the Jacobian matrix can be obtained by differentiating the direct geometric model $X=J(\theta)$ with respect to joint position $\theta$ as:

$$
J_{i j}=\frac{\partial f_{i}(\theta)}{\partial \theta_{j}}
$$

In order to compute the Jacobian matrix, using the DH frames, i.e.

$$
\begin{aligned}
J_{v}(\theta) & =\left\{\begin{array}{c}
\mathbf{Z}_{0}^{\mathbf{i}-1},: \text { for prismatic joint } \\
\mathbf{Z}_{0}^{\mathbf{i}-1} *\left[\mathbf{0}_{0}^{\mathbf{i}}-\mathbf{0}_{0}^{\mathrm{i}-1}\right]: \text { for revolute joint }
\end{array}\right. \\
J_{\omega}(\boldsymbol{\theta}) & =\left\{\begin{array}{c}
\mathbf{0},: \text { for prismatic joint } \\
\mathbf{Z}_{0}^{\mathrm{i}-1}: \text { for revolute joint }
\end{array}\right.
\end{aligned}
$$

The Jacobian matrix is formulated in the form of

$$
\begin{aligned}
& J_{v}(\theta)_{(3 \times 6)} \\
& J_{\omega}(\theta)_{(3 \times 6)}
\end{aligned}
$$

$$
\begin{gathered}
=R_{0}^{1}\left[\begin{array}{l}
0 \\
0 \\
1
\end{array}\right] X\left(P_{0}^{6}-P_{0}^{1}\right) \quad R_{0}^{2}\left[\begin{array}{l}
0 \\
0 \\
1
\end{array}\right] X\left(P_{0}^{6}-P_{0}^{2}\right) \\
R_{0}^{3}\left[\begin{array}{l}
0 \\
0 \\
1
\end{array}\right] X\left(P_{0}^{6}-P_{0}^{3}\right) \quad R_{0}^{4}\left[\begin{array}{l}
0 \\
0 \\
1
\end{array}\right] X\left(P_{0}^{6}-P_{0}^{4}\right) \\
R_{0}^{5}\left[\begin{array}{l}
0 \\
0 \\
1
\end{array}\right] X\left(P_{0}^{6}-P_{0}^{5}\right) \quad R_{0}^{6}\left[\begin{array}{l}
0 \\
0 \\
1
\end{array}\right]
\end{gathered}
$$

$R_{0}^{1}\left[\begin{array}{l}0 \\ 0 \\ 1\end{array}\right] \quad R_{0}^{2}\left[\begin{array}{l}0 \\ 0 \\ 1\end{array}\right] \quad R_{0}^{3}\left[\begin{array}{l}0 \\ 0 \\ 1\end{array}\right]$

$R_{0}^{4}\left[\begin{array}{l}0 \\ 0 \\ 1\end{array}\right] \quad R_{0}^{5}\left[\begin{array}{l}0 \\ 0 \\ 1\end{array}\right] \quad R_{0}^{6}\left[\begin{array}{l}0 \\ 0 \\ 0\end{array}\right]$.

Similarly, the acceleration can be obtained by differentiating the velocity equations as:

$$
\begin{gathered}
\frac{d}{d t}(\dot{X})=\frac{d}{d t}(J(\theta) \dot{\boldsymbol{\theta}}) \\
\ddot{X}=J(\theta) \ddot{\boldsymbol{\theta}}+\left(\frac{\boldsymbol{d}}{\boldsymbol{d} \boldsymbol{t}} J(\theta)\right) \dot{\boldsymbol{\theta}}
\end{gathered}
$$

\section{Dynamic analysis of a walking machine leg mechanism}

In the study of the dynamics of leg mechanisms, the forces and/or torques required to cause motion of the mechanisms are considered to derive the kinematics of the leg mechanisms or suspension systems using DH convention approach (as discussed in the previous section) without consideration of the forces and moments producing the motion. Under this section, the dynamic analysis is carried out to formulate the parametric equation of motion which describes the relationship between force and motion.

The dynamics of mechanisms can be obtained in various ways, namely by using a Newton-Euler dynamic formulation, a Lagrangian formulation, Kane's Method, and the like. The Newton-Euler method is based on Newton's second law of motion with its rotational analog called Euler's equation. It describes how forces and moments 
are related to acceleration. In the iterative Newton-Euler algorithm, the position, velocity and acceleration of the joints are known. Using these parameters as input and assuming that the mass properties of the mechanism and any externally acting forces are known, the joint torques required to cause this motion can be calculated.

Dynamics of the walking machine can be divided into two basic categories: forward and inverse dynamics. The forward dynamics deals with finding the response of a given rigid body influenced by force and torques applied on it, which was simulated by providing link length and rotating angle to determine the position [14]. On the other hand, the opposite procedure is implemented to find the force and torques where motion is created in the system through inverse dynamic analysis. The link length and position is given to determine the angle of rotation of the links. This method is widely used in the control system of a motion [17]. In general, for the analysis and modeling of dynamic equations of complex mechanisms in robot design subjected to holonomic constraints, the Newton-Euler and EulerLagrange formulations are most common ones. The Lagrangian equation formulation involves the kinetic and potential energy of the system.

For the walking machine, the kinematic motion analysis is of the leg mechanism and the consideration of the equation of motion is crucial. In order to perform forward dynamic analysis, some assumptions need to be made, including the following.

1. The links are rigid.

2. The friction in the joints is ignored.

3. The leg mechanism is assumed to be at a constant velocity with no inclination terrain.

4. When the leg is in contact with the ground, it is assumed to have zero impact.

Furthermore, the generic dynamic equation can be formulated as an equation of motion for the leg mechanism.

\section{Euler-Lagrange equation of the leg mechanism}

Let $\theta_{1}, \ldots . ., \theta_{1}$ be generalized coordinates that completely locate a dynamic system. Let $K$ and $V$ be the total kinetic and potential energy stored in a dynamic system, respectively. The Lagrange method is based on describing the scalar energy functions of the system, including the kinetic energy $K(\theta, \dot{\theta})$ and the potential energy $V(\theta)$. The two energy functions can be expressed in terms of the joint positions $\theta$ and the joint velocities $\dot{\theta}$.

Lagrange equation can be defined as:

$$
\left(\theta_{i}, \dot{\theta}_{l}\right)=K-V
$$

Since the kinetic and potential energies are functions of $\theta_{i}$ and $\dot{\theta}_{l},(i=1,2,3, \ldots n)$ using the Lagrangian equation of motion, the dynamic system is given by

$$
\frac{d}{d t} \frac{\partial L}{\partial \dot{\theta}_{l}}-\frac{\partial L}{\partial \theta_{i}}=Q_{i}
$$

where: $Q_{i}=$ externally applied generalized force,

$L=K-V$,

$V=V(\theta)$ and is the potential energy,

$K=K(\theta)$ is the kinetic energy, and

$\mathrm{i}=1, \ldots, n$.

\section{Kinetic and potential energy expression}

In order to use the Euler Lagrange equations, the kinetic and potential energy has to be expressed for the leg mechanisms. After the derivation of kinetic and potential energy for each link, the Lagrangian of the leg mechanisms is the summation of the individual Lagrangians. The overall kinetic energy is given by:

$$
\begin{aligned}
K=K_{t}+K_{r} & =\frac{1}{2} m \dot{v}^{T} \dot{v}+\frac{1}{2} I \dot{\omega}^{T} \dot{\omega} \\
K_{i} & =\frac{1}{2} v^{T} M v
\end{aligned}
$$

For $n$ linkages, the kinetic energy expression can be expressed as

$$
K=\sum_{i=1}^{n} K_{i}=\frac{1}{2} \sum_{i=1}^{n}\left(v^{T} M v\right)_{i}
$$

This becomes

$$
K=\sum_{i=1}^{n} \frac{1}{2} J(\theta) \dot{\theta}^{T} M J(\theta) \dot{\theta}=\frac{1}{2} \dot{\theta}^{T} M(\theta) \dot{\theta}
$$

where: $M(\theta)=\sum_{i=1}^{n} J(\theta)^{T} M J(\theta)$, in which $M$ is a generalized inertial matrix of mass and moment of inertia, symmetric and positive definite matrix.

The kinematic properties of the rigid body are fully described by its mass, principal axis and 
moments of inertia. Inertia tensor $I_{J}$ can be made diagonal as

$$
\begin{aligned}
& I_{J}=R I R^{T}=\left[\begin{array}{lll}
I_{x x} & I_{x y} & I_{x z} \\
I_{y x} & I_{y y} & I_{y z} \\
I_{z x} & I_{z y} & I_{z z}
\end{array}\right] \\
& \text { and } M=\left[\begin{array}{lll}
m_{x x} & m_{x y} & m_{x z} \\
m_{y x} & m_{y y} & m_{y z} \\
m_{z x} & m_{z y} & m_{z z}
\end{array}\right]
\end{aligned}
$$

where: $R$ is a rotational matrix of the homogenous transformation matrix

Considering the coordinate axis and principal axis aligned together, the inertial tensor would be only diagonal:

$$
M(\theta)=\left[\begin{array}{cccccc}
m_{x x} & 0 & 0 & 0 & 0 & 0 \\
0 & m_{y y} & 0 & 0 & 0 & 0 \\
0 & 0 & m_{z z} & 0 & 0 & 0 \\
0 & 0 & 0 & I_{x x} & 0 & 0 \\
0 & 0 & 0 & 0 & I_{y y} & 0 \\
0 & 0 & 0 & 0 & 0 & I_{z z}
\end{array}\right]
$$

By summing the translational and rotational kinetic energy of the link,

$$
K=\sum_{i=1}^{n}\left(\frac{1}{2} m_{i} \dot{v}_{i}^{T} \dot{v}_{i}+\frac{1}{2} I_{i} \dot{\omega}_{i}{ }^{T} \dot{\omega}_{i}\right)
$$

where: $\dot{x}$ denotes the velocity of the center of mass of the rigid link,

$\dot{\omega}$ is the angular velocity vector and

$I$ is the inertia matrix.

In the case of the rigid body, gravity is the source of potential energy, due to the mass of the links. In most cases, the potential energy is defined along the unit vector acting through the center of mass of each link. The potential energy is expressed as:

$$
V(\theta)=\sum_{i=1}^{n} m_{i} g^{T} h_{i}(\theta)
$$

In Eq. 30 and 31 we have computed an expression for kinetic and potential energy given in respectively

$$
\begin{aligned}
& \mathcal{L}\left(\theta_{i}, \dot{\theta}_{l}\right)=K-V \\
& \mathcal{L}\left(\theta_{i}, \dot{\theta}_{l}\right)=\frac{1}{2} \dot{\theta}^{T} M(\theta) \dot{\theta}-V(\theta)
\end{aligned}
$$

Using the Euler Lagrange equations that describe the dynamics for each of the generalized coordinates, recalling the equations and inserting them into the Lagrange equation given in Eq. 23 as well as taking its derivative required considering that the potential energy does not depend on $\dot{\theta}$. yields [18]:

$$
M(\theta) \ddot{\theta}+C(\theta, \dot{\theta}) \dot{\theta}+G(\theta)=\tau_{i}
$$

where $M$ and $C$ represent inertial properties (inertial matrix and Coriolis effect), and $G$ represents gravity terms.

\section{DISCUSSION OF RESULTS}

This chapter discusses the results obtained to determine the effects of the kinematic behavior in motion analysis tool of the leg mechanism responses. The CAD geometry of the walking machine was investigated in SolidWorks kinematic motion analysis. The data were exported to Excel and interpreted with MATLAB software. The results obtained from the kinematic motion analysis were explained briefly.

\section{Kinematic motion analysis in SolidWorks}

A kinematic motion analysis is the imitation of the operation of a real-world process or system over time. It is a tool to evaluate the kinematic response of a system, existing or proposed, under different configurations of interest and over long periods of real time. The behavior of a system that evolves over time is studied by developing a kinematic model. The motion analysis consists of building a computer model that describes the behavior of a system with which system exploration and analysis that supports decisions are conducted. The mathematical model is used to determine the response of the system in different situations using one of the motion analysis functions available in SolidWorks such as animation, kinematic analysis and motion analysis functionalities.

Motion analysis is the most sophisticated analysis functions reflecting all the required features such as inertial properties, external forces, contacts, mate friction etc. [19]. The kinematic analysis is performed to determine the displacement, velocity, acceleration and torque responses. MATLAB 2016a was used to analyze the data obtained by importing and performing the leg responses in plots. The motion analysis is performed based on constant input functions and fluctuating input functions as flat and rough terrain, respectively. 


\section{Motion analysis of walking machine leg mechanism}

SolidWorks motion analysis was used to determine the response of the walking machine leg in terms of displacement, velocity and acceleration based on the input provided. The response of the single leg in Figure 2(a) shows the smooth profile of its kinematic behavior with no external input function. As the displacement increases or decreases, both the velocity and acceleration proportionally change. Theoretically, it was proven that the relationships between displacement, velocity and acceleration are directly proportional and time-dependent.

Figure 2(b) indicates that the generated torque profile shows the graph is smooth and repeats the same path over motion patterns. The degree of smoothness can determine the behavior of the motor that will be used in the system. As the smoothness of the torque graph decreases, the motor exhibits unwanted motion fluctuation that can damage the motor. Thus, generating the torque profile graph is basically used to determine the specification of the motor to be used. The maximum torque recorded here is $0.3 \mathrm{Nmm}$ and minimum of $0.17 \mathrm{Nmm}$ within the initial boundary considered in this motion analysis. The peak value obtained in the motion analysis describes the point at which high torque is required. On the basis of the mechanism, when the leg mechanism starts to move up while rotating, it needs a large amount of torque.

a)

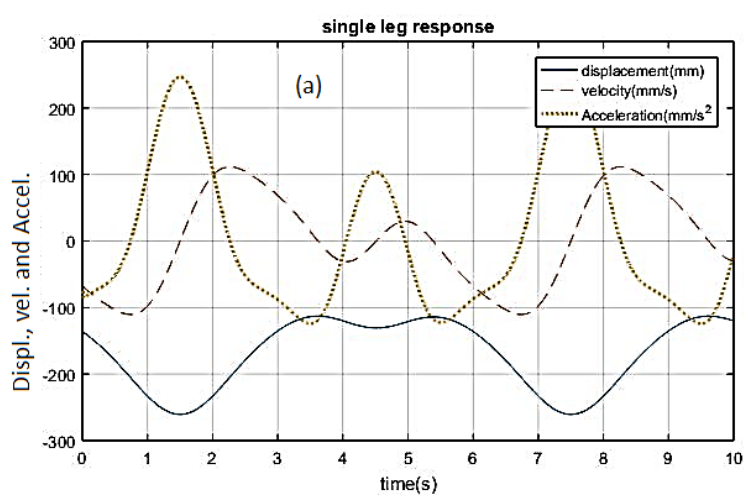

In the motion analysis, the derived equation of motion parameters was considered to show the responses. These parameters included the gravitational energy, the mass matrix from the property of material assigned to it in modeling, the external force as input from the motor and the properties of rough terrain as an external input, which affect the motion of the walking machine legs.

Figure 3 shows the motion analysis results considering the fluctuating input function from the ground by means of force. From this figure, it is possible to observe that as the amplitude of the input force fluctuates, the torque required to drive the mechanism against the force varies. This can be theoretically validated because torque and force have a direct relationship.

Furthermore, the comparison between constant input function and fluctuating input function was shown in Figure 4 in terms of displacement, velocity, acceleration and torque responses. Generally, with various terrain parameters, the leg mechanism behaves differently and gives different responses. This is shown clearly in the motion analysis result from a constant input function and fluctuating input function, Figure 2 and 3 respectively. On rough terrain, the displacement, velocity and acceleration obtained take time when compared to the flat surface. This shows that the performance of the walking machine can be affected by the terrain topology. As the amplitude of the rough terrain variation increases, the performance of the walking machine can be affected even more.

b)

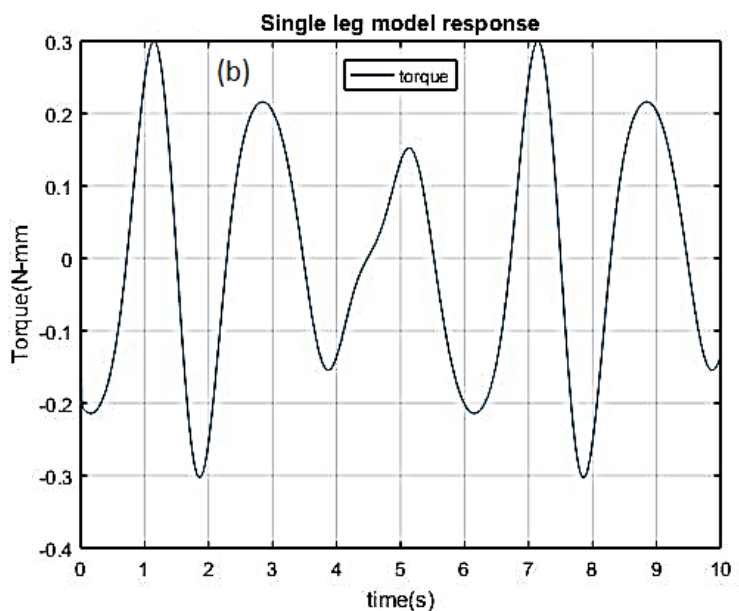

Fig. 2. Leg mechanism response of walking machine (a) Displacement, velocity and acceleration response, (b) Torque profile 
a)

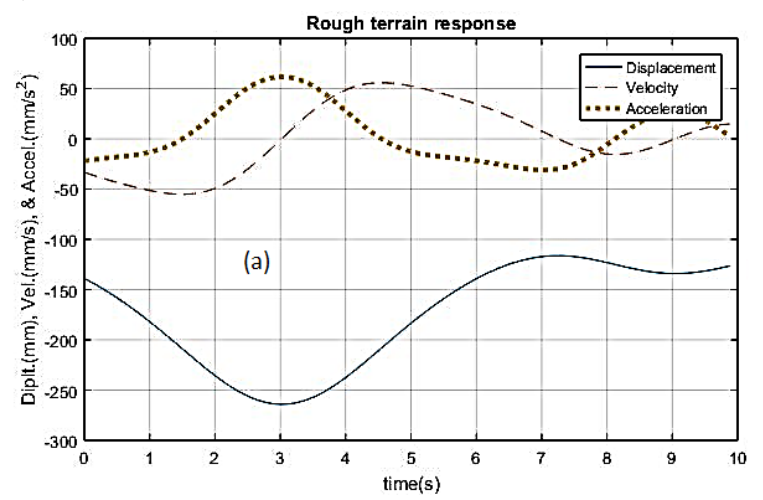

b)

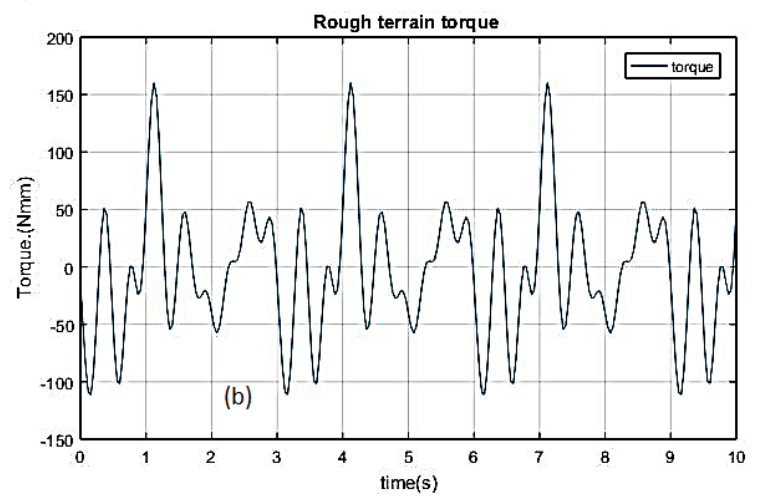

Fig. 3. Leg mechanism response of walking machine (a) Displacement, velocity and acceleration response, (b) Torque response of fluctuating input function

a)

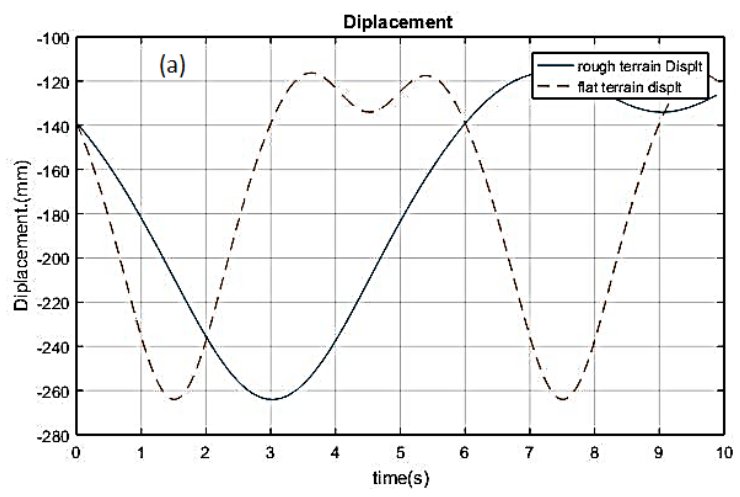

b)

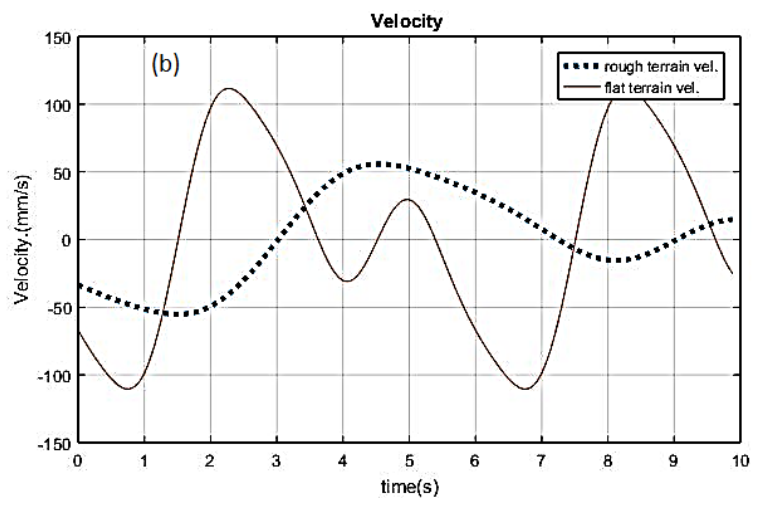

c)

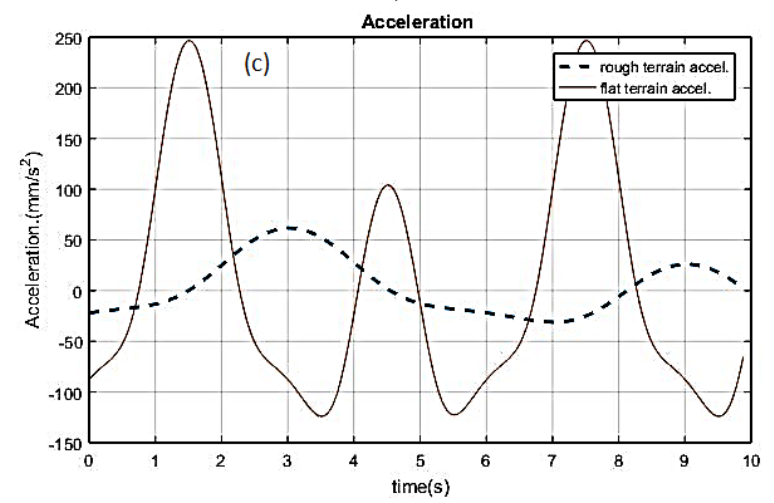

Fig. 4. Comparison between constant and fluctuating input function (a) Displacement comparison,

(b) Velocity comparison, (c) Acceleration response

\section{CONCLUSIONS}

In the study partly reported in this article, various previous works on the topic were reviewed and it was found that the leg mechanism is a comparatively better solution for rough terrain over wheels or track mechanism. The mechanism of the leg is modeled kinematically using integration of linkages without multiple uses of motors at each actuating DOF. This reduced the number of motors, which has a great effect on the energy consumption. The leg mechanism developed also enables to walk on rough terrain while maintaining static stability. A low number of components and simple design ensures minimum cost for the machine to be manufactured.

From kinematic synthesis and analysis, dimensional synthesis was parametrically derived for the leg mechanisms using forward kinematic and inverse kinematic and the position analysis 
were carried out in a vector form. The DH convention approach was applied to analyze the mechanisms using the Transformation matrix for the formulated relation between position, velocity and acceleration analysis using the Jacobian Matrix. The dynamic analysis was carried out using Euler-Lagrange method by considering the kinetic energy and potential energy expression and then the equation of motion was derived.

The part and assembly geometric modeling was conducted in SolidWorks V2018 and the motion analysis results were exported and analyzed in MATLAB 2016a, in which the plots of displacement, velocity, acceleration and torque responses were generated. On the basis of the responses observed from the effect of variation of the rough terrain on the kinematic behavior of the leg mechanism, it was concluded that the used special type of the four-bar linkage mechanism is well-suited for the walking of the machine on rough terrain. The proposed and employed simple linkage integration also enabled the synthesis of the mechanism and generation of the motion paths. It was further concluded that the parametric equation helps to derive scalable design to any size for constructing the machine. Finally, the design with reduced number of actuator is a significant factor for reducing the energy consumption.

\section{REFERENCES}

1. Roennau, A., Kerscher, T. and Dillmann, R., Design and kinematics of a biologically-inspired leg for a six-legged walking machine, Proc. of the 2010 3rd IEEE RAS \& EMBS Int. Conference on Biomedical Robotics and Biomechatronics, The University of Tokyo, Japan, Sept. 26-29, 2010.

2. Berns, K., Ilg, W., Deck, M., Albiez, J. and Dillmann, R., Mechanical construction and computer architecture of the four-legged walking machine BISAM, IEEE/ASME Transaction on mechatronics, 4(1), 1999, 32-38.

3. Collins, S.H., Wisse, M. and Ruina, A., A threedimensional passive-dynamic walking robot with two legs and knees, The Int. Journal of Robotics Research, 20(7), 2001, 607-615.

4. Sosnowski, M. and Jaskowski, J., Didactic automated station of complex kinematics, Advances in
Science and Technology Research Journal, 8(21), 2014, 18-23, DOI: 10.12913/22998624.1091873.

5. McCarthy, J.M., Kinematics, polynomials, and computer: A brief history, Journal of Mechanisms Robotics 3(1), 2011, 010201, doi:10.1115/1.4003039.

6. Moon, F. C., History of the dynamics of machines and mechanisms from Leonardo to Timoshenko, Int. Symposium on History of Machines and Mechanisms , H.S.Yan and M.Ceccarelli, Eds. 2009

7. Denavit, J. and Hartenberg, R.S., Kinematic synthesis of linkages, McGraw-Hill, Inc. NY, 1964.

8. Natesan, A.K., Kinematic analysis and synthesis of four-bar mechanisms for straight line coupler curves: Rochester Institute of Technology, 1994.

9. Myszka, D.H., Machines and mechanisms: Applied kinematics analysis, 4th ed., Pearson Education Inc. NJ, 2012.

10. Khurimi, R.S. and Gupta, J.K. Theory of machines. 8th ed., S. Chand \& Company Ltd. 2005, 1070.

11. Ravani, B., Kinematics and mechanisms. In: Richard C., Ed. Engineering Handbook California: CRC Press LLC, 2000.

12. Waldron, K.J. and Kinzel, G.L., Kinematics, dynamics, and design of machinery. 2nd ed., Wiley \& Sons, 2001.

13. Sandor, G.N. and Erdman, A.G., Advanced mechanism analysis, Prentice-Hall Int. Inc. NJ, 1984. 701.

14. Manjaree, S., Kinematics and dynamics of multidegree of freedom robotic systems using analytical and artificial intelligent techniques with experimental validation, The Northcap University, 2016.

15. Spong, M.W., Hutchinson, S. and Vidyasagar, M., Robot dynamics and control. 2nd ed. 2004.

16. Harrison, H.R. and Nettleto, T., Advanced engineering dynamics. Britain John Wiley \& Sons Inc.; 1997.

17. Yang, Ch., Ma, H. and Fu, M., Robot kinematics and dynamics modeling. Advanced Technologies in Modern Robotic Applications. Singapore: Press and Springer Science+Business Media, 2016.

18. Tresnak, B.A., Forces acting on the robot during grinding. Prague, Czech Technical University, 2016.

19. Siciliano, B., Sciavicco, L., Villani, L. and Oriolo, G., Robotics modeling, planning and control, Italy: Springer, 2009.

20. Toreh, E.H., Shahmohammadi, M. and Khamseh, N., Kinematic and kinetic study of rescue robot by SolidWorks software. Research Journal of Applied Sciences, Engineering and Technology, 5(21), 2013, 5070-6. 